International Journal of Pure and Applied Mathematics

Volume 87 No. 1 2013, 129-143

ISSN: 1311-8080 (printed version); ISSN: 1314-3395 (on-line version)

url: http://www.ijpam.eu

doi: http://dx.doi.org/10.12732/ijpam.v87i1.7

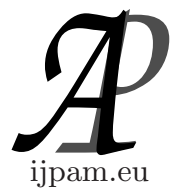

\title{
A FAMILY OF $p$-VALENT ANALYTIC FUNCTIONS DEFINED BY A FRACTIONAL CALCULUS OPERATOR
}

\author{
Mamta Pathak ${ }^{1}$, Poonam Sharma ${ }^{2}$ \\ Babu Banarasi Das Group of Educational Institutions \\ Faizabad Road, Lucknow, INDIA \\ ${ }^{2}$ Deparment of Mathematics and Astronomy \\ University of Lucknow \\ Lucknow, INDIA
}

\begin{abstract}
In this Paper a family $S(\alpha, \beta, \mu, p)$ of $p$-valent analytic functions involving fractional calculus operator $\Omega_{z}^{\mu, p}$ is studied and a sufficient coefficient condition for functions belonging to the family $S(\alpha, \beta, \mu, p)$ is proved and it is shown that this coefficient condition is necessary for its subfamily $T S(\alpha, \beta, \mu, p)$. Coefficient estimate, growth theorem and results on partial sums are obtained for the family $S(\alpha, \beta, \mu, p)$. Also an integral inequality is proved for functions belonging to the family $T S(\alpha, \beta, \mu, p)$.
\end{abstract}

AMS Subject Classification: 30C45, 30C55

Key Words: analytic functions, starlike function, convex functions, partial sums, integral mean inequality

\section{Prelimnaries}

Let $A(p)$ denotes a family of functions of the form:

$$
f(z)=z^{p}+\sum_{k=1}^{\infty} a_{p+k} z^{p+k}(p \in N=\{1,2,3 \ldots .\})
$$

which are analytic and $p$-valent in the open unit disk $U=\{z: z \in C$ and $|z|<$

Received: May 12, 2013

(C) 2013 Academic Publications, Ltd.

$\S$ Correspondence author url: www.acadpubl.eu 
1 ) and $T(p)$ denotes a subfamily of $A(p)$ whose members are of the form:

$$
f(z)=z^{p}-\sum_{k=1}^{\infty}\left|a_{p+k}\right| z^{p+k}(p \in N=\{1,2,3 \ldots\}) .
$$

Denote $A(1) \equiv S$.

The convolution or Hadamard product of $f(z)$ given by $(1)$ and $g(z) \in A(p)$ given by

$$
g(z)=z^{p}+\sum_{k=1}^{\infty} b_{p+k} z^{p+k}
$$

is defined as:

$$
(f * g)(z)=z^{p}+\sum_{k=1}^{\infty} a_{p+k} b_{p+k} z^{p+k}
$$

which is analytic and $p$-valent in the unit disk $U$.

Let $S^{*}(p, \alpha)$ and $K(p, \alpha)$ denote respectively the family of starlike and convex functions $f(z) \in A(p)$ satisfying

$$
\operatorname{Re}\left\{\frac{z f^{\prime}(z)}{f(z)}\right\}>\alpha \text { and } \operatorname{Re}\left\{1+\frac{z f^{\prime \prime}(z)}{f^{\prime}(z)}\right\}>\alpha, 0 \leq \alpha<p
$$

respectively. Again, let $\beta-U S T(\alpha, p)$ and $\beta-U C V(\alpha, p)$ denote respectively the family of $\beta$-uniformly starlike and $\beta$-uniformly convex functions $f(z) \in A(p)$ satisfying

$$
\operatorname{Re}\left\{\frac{z f^{\prime}(z)}{f(z)}\right\}>\beta\left|\frac{z f^{\prime}(z)}{f(z)}-p\right|+\alpha
$$

and

$$
\operatorname{Re}\left\{1+\frac{z f^{\prime \prime}(z)}{f^{\prime}(z)}\right\}>\beta\left|1+\frac{z f^{\prime \prime}(z)}{f^{\prime}(z)}-p\right|+\alpha
$$

respectively for some $\beta \geq 0,0 \leq \alpha<p$ and $z \in U$. Clearly $0-U S T(\alpha, p) \equiv$ $S^{*}(p, \alpha), 0-U C V(\alpha, p) \equiv K(p, \alpha)$. Denote $S^{*}(1, \alpha) \equiv S^{*}(\alpha), K(1, \alpha) \equiv K(\alpha)$ and $\beta-U S T(\alpha, 1) \equiv \beta-U S T(\alpha), \beta-U C V(\alpha, 1) \equiv \beta-U C V(\alpha)$.

Saitoh [8] introduced a Carlson-Shaffer type operator $L_{p}(a, c)$ for $f(z) \in$ $A(p)$ which is defined as:

$$
L_{p}(a, c) f(z):=\phi_{p}(a, c, z) * f(z)
$$

where $\phi_{p}(a, c, z)$ is defined as: 


$$
\phi_{p}(a, c, z)=z^{p}+\sum_{k=1}^{\infty} \frac{(a)_{k}}{(c)_{k}} z^{p+k}
$$

for $a \in R_{+}, c \in R / Z_{0}^{-}=\{0,-1,-2,-3 \ldots \ldots \ldots\}$ and $(a)_{k}$ is the Pochhammer symbol defined as:

$$
(a)_{k}=\frac{\Gamma(a+k)}{\Gamma(a)}=a(a+1)(a+2) \ldots(a+k-1), k \in N=1,2, \ldots
$$

The Riemann-Liouville fractional derivative operator [9] of order $\mu(0 \leq \mu \leq$ 1) for analytic function $f(z)$ is defined as:

$$
\begin{gathered}
D_{z}^{\mu} f(z)=\frac{1}{\Gamma(1-\mu)} \frac{d}{d z} \int_{0}^{z} \frac{f(t)}{(z-t)^{\mu}} d t, \quad 0 \leq \mu<1 \\
D_{z}^{1} f(z)=f^{\prime}(z)
\end{gathered}
$$

which is analytic in simply connected region of $z$-plane containing the origin, multiplicity of $(z-t)^{\mu}$ is removed by taking $\log (z-t)$ to be real when $(z-t)>0$ and is well defined in the unit disk $U$.

The image of power function $z^{k}$ under the operator defined in (10) is given as:

$$
D_{z}^{\mu}\left(z^{k}\right)=\frac{\Gamma(k+1)}{\Gamma(k-\mu+1)} z^{k-\mu}, \quad 0 \leq \mu \leq 1 .
$$

Thus, the normalized operator $\Omega_{z}^{\mu, p}: A(p) \rightarrow A(p)$ is defined as:

$$
\Omega_{z}^{\mu, p} f(z)=z^{\mu} \frac{\Gamma(p+1-\mu)}{\Gamma(p+1)} D_{z}^{\mu} f(z), 0 \leq \mu \leq 1, p \in N
$$

and its series expansion using (12) for $f(z) \in A(p)$ of the form (1) is given as:

$$
\Omega_{z}^{\mu, p} f(z)=z^{p}+\sum_{k=1}^{\infty} a_{p+k} \phi_{p}^{\mu}(k) z^{p+k}
$$

where

$$
\phi_{p}^{\mu}(k)=\frac{(p+1)_{k}}{(p+1-\mu)_{k}} .
$$

Involving operator $\Omega_{z}^{\mu, p}$ given in (13), a generalized family $S(\alpha, \beta, \mu, p)$ of functions $f(z) \in A(p)$ for some $\beta \geq 0,0 \leq \alpha<p, 0 \leq \mu \leq 1$ is defined by

$$
\operatorname{Re}\left\{\frac{z\left(\Omega_{z}^{\mu, p} f(z)\right)^{\prime}}{\Omega_{z}^{\mu, p} f(z)}\right\}>\beta\left|\frac{z\left(\Omega_{z}^{\mu, p} f(z)\right)^{\prime}}{\Omega_{z}^{\mu, p} f(z)}-p\right|+\alpha .
$$


It is examined that if $f(z) \in S(\alpha, \beta, \mu, p)$ with $0 \leq \beta \leq \frac{\alpha}{p}, 0 \leq \alpha<p$, then

$$
\Omega_{z}^{\mu, p} f(z) \in S^{*}\left(p, \frac{\alpha-\beta p}{1-\beta}\right)
$$

Note that $S(\alpha, \beta, 0, p) \equiv \beta-U S T(\alpha, p)$ and $S(\alpha, \beta, 1, p) \equiv \beta-U C V(\alpha, p)$. Also, $T S(\alpha, \beta, \mu, p) \equiv S(\alpha, \beta, \mu, p) \cap T(p)$.

Further, it is observed that $S(\alpha, 0, \mu, p) \equiv S_{\mu}(\alpha, p, 1,-1)$, a family studied in chapter 2 .

\section{Coefficient Conditions}

In this section, a sufficient coefficient condition for functions belonging to $S(\alpha, \beta, \mu, p)$ family is given and then it is proved that this condition is necessary for its subfamily $T S(\alpha, \beta, \mu, p)$.

Theorem 1. Let $f(z) \in A(p)$ of the form (1), satisfies

$$
\sum_{k=1}^{\infty} \frac{\{k(1+\beta)+(p-\alpha)\}}{(p-\alpha)} \phi_{p}^{\mu}(k)\left|a_{p+k}\right| \leq 1
$$

for $\beta \geq 0,0 \leq \alpha<p, 0 \leq \mu \leq 1, \phi_{p}^{\mu}(k)=\frac{(p+1)_{k}}{(p+1-\mu)_{k}}$, then $f(z) \in S(\alpha, \beta, \mu, p)$.

Proof. Let the inequality (17) holds true, then it is to prove that

$$
\operatorname{Re}\left\{\frac{z\left(\Omega_{z}^{\mu, p} f(z)\right)^{\prime}}{\Omega_{z}^{\mu, p} f(z)}-p\right\}>\beta\left|\frac{z\left(\Omega_{z}^{\mu, p} f(z)\right)^{\prime}}{\Omega_{z}^{\mu, p} f(z)}-p\right|+\alpha-p
$$

or,

$$
\beta\left|\frac{z\left(\Omega_{z}^{\mu, p} f(z)\right)^{\prime}}{\Omega_{z}^{\mu, p} f(z)}-p\right|-\operatorname{Re}\left\{\frac{z\left(\Omega_{z}^{\mu, p} f(z)\right)^{\prime}}{\Omega_{z}^{\mu, p} f(z)}-p\right\}<p-\alpha .
$$

Since, $\operatorname{Re}(w)$ or $\operatorname{Re}(-w) \leq|w|$ for some complex number $w$, it follows that

$$
\begin{gathered}
\beta\left|\frac{z\left(\Omega_{z}^{\mu, p} f(z)\right)^{\prime}}{\Omega_{z}^{\mu, p} f(z)}-p\right|-\operatorname{Re}\left\{\frac{z\left(\Omega_{z}^{\mu, p} f(z)\right)^{\prime}}{\Omega_{z}^{\mu, p} f(z)}-p\right\} \\
\leq(1+\beta)\left|\frac{z\left(\Omega_{z}^{\mu, p} f(z)\right)^{\prime}}{\Omega_{z}^{\mu, p} f(z)}-p\right|
\end{gathered}
$$




$$
\leq(1+\beta) \frac{\sum_{k=1}^{\infty} k \phi_{p}^{\mu}(k)\left|a_{p+k}\right|}{1-\sum_{k=1}^{\infty} \phi_{p}^{\mu}(k)\left|a_{p+k}\right|}
$$

whose right hand side expression is bounded above by $(p-\alpha)$ if, inequality (17) holds, which proves Theorem 1

Theorem 2. Let $f(z) \in T(p)$ be of the form (2), then $f(z) \in T S(\alpha, \beta, \mu, p)$ if and only if

$$
\sum_{k=1}^{\infty} \frac{\{k(1+\beta)+(p-\alpha)\}}{(p-\alpha)} \phi_{p}^{\mu}(k)\left|a_{p+k}\right| \leq 1,
$$

for $\beta \geq 0,0 \leq \alpha<p, 0 \leq \mu \leq 1$ and $\phi_{p}^{\mu}(k)=\frac{(p+1)_{k}}{(p+1-\mu)_{k}}$.

Proof. In view of Theorem 1, it needs only to prove necessary part. Let $f(z) \in T S(\alpha, \beta, \mu, p)$, then $f(z)$ of the form (2) satisfies

$$
\operatorname{Re}\left\{\frac{z\left(\Omega_{z}^{\mu, p} f(z)\right)^{\prime}}{\Omega_{z}^{\mu, p} f(z)}\right\}>\beta\left|\frac{z\left(\Omega_{z}^{\mu, p} f(z)\right)^{\prime}}{\Omega_{z}^{\mu, p} f(z)}-p\right|+\alpha
$$

or,

$$
\begin{aligned}
& \beta\left|\frac{p-\sum_{k=1}^{\infty}(p+k) \phi_{p}^{\mu}(k)\left|a_{p+k}\right| z^{k}}{1-\sum_{k=1}^{\infty} \phi_{p}^{\mu}(k)\left|a_{p+k}\right| z^{k}}-p\right| \\
& <\operatorname{Re}\left\{\frac{p-\sum_{k=1}^{\infty}(p+k) \phi_{p}^{\mu}(k)\left|a_{p+k}\right| z^{k}}{1-\sum_{k=1}^{\infty} \phi_{p}^{\mu}(k)\left|a_{p+k}\right| z^{k}}-\alpha\right\} .
\end{aligned}
$$

Since, $\operatorname{Re}(w)$ or $\operatorname{Re}(-w) \leq|w|$,

$$
\begin{aligned}
& \operatorname{Re} \beta\{p\left.-\frac{p-\sum_{k=1}^{\infty}(p+k) \phi_{p}^{\mu}(k)\left|a_{p+k}\right| z^{k}}{1-\sum_{k=1}^{\infty} \phi_{p}^{\mu}(k)\left|a_{p+k}\right| z^{k}}\right\} \\
&<\operatorname{Re}\left\{\frac{p-\sum_{k=1}^{\infty}(p+k) \phi_{p}^{\mu}(k)\left|a_{p+k}\right| z^{k}}{1-\sum_{k=1}^{\infty} \phi_{p}^{\mu}(k)\left|a_{p+k}\right| z^{k}}-\alpha\right\} .
\end{aligned}
$$

Letting $z \rightarrow 1^{-}$along the real axis, it gives the desired inequality

$$
\sum_{k=1}^{\infty} \frac{\{k(1+\beta)+(p-\alpha)\}}{(p-\alpha)} \phi_{p}^{\mu}(k)\left|a_{p+k}\right| \leq 1 .
$$

This proves Theorem 3 . 


\section{Coefficient Estimate for the Family $S(\alpha, \beta, \mu, p)$}

Theorem 3. Let $f(z) \in A(p)$ of the form (1) be in the family $S(\alpha, \beta, \mu, p)$, $0 \leq \beta \leq \frac{\alpha}{p}, 0 \leq \alpha<p$, then

$$
\left|a_{p+k}\right| \leq \frac{1}{k ! \phi_{p}^{\mu}(k)} \prod_{j=1}^{k}\left(j-1+\frac{2(p-\alpha)}{(1-\beta)}\right), k \geq 1
$$

where $\phi_{p}^{\mu}(k)=\frac{(p+1)_{k}}{(p+1-\mu)_{k}}$.

Or, equivalently,

$$
\left|a_{p+k}\right| \leq \frac{\left(\frac{2(p-\alpha)}{1-\beta}\right)_{k}(p+1-\mu)_{k}}{(1)_{k}(p+1)_{k}}, k \geq 1
$$

Proof. Since $f(z) \in S(\alpha, \beta, \mu, p)$ for $0 \leq \beta \leq \frac{\alpha}{p}, 0 \leq \alpha<p$, it gives

$$
\operatorname{Re}\left\{\frac{z\left(\Omega_{z}^{\mu, p} f(z)\right)^{\prime}}{\Omega_{z}^{\mu, p} f(z)}\right\}>\frac{(\alpha-\beta p)}{(1-\beta)} .
$$

Let $q(z)=1+q_{1} z+q_{2} z^{2}+\ldots$, be defined as

$$
q(z)=\frac{(1-\beta)\left\{\frac{z\left(\Omega_{z}^{\mu, p} f(z)\right)^{\prime}}{\Omega_{z}^{\mu, p} f(z)}\right\}-(\alpha-\beta p)}{(p-\alpha)}
$$

which is analytic in $U$ with $q(0)=1$ and $\operatorname{Re}\{q(z)\}>0$ for $z \in U$, then

$$
z\left(\Omega_{z}^{\mu, p} f(z)\right)^{\prime}-\frac{(\alpha-\beta p)}{(1-\beta)} \Omega_{z}^{\mu, p} f(z)=\frac{(p-\alpha)}{(1-\beta)} q(z) \Omega_{z}^{\mu, p} f(z) .
$$

On writing their respective series expansions, it gives

$$
\begin{gathered}
z\left(p z^{p-1}+\sum_{k=1}^{\infty}(p+k) \phi_{p}^{\mu}(k) a_{p+k} z^{p+k-1}\right)-\frac{(\alpha-\beta p)}{(1-\beta)}\left(z^{p}+\sum_{k=1}^{\infty} \phi_{p}^{\mu}(k) a_{p+k} z^{p+k}\right) \\
=\frac{(p-\alpha)}{(1-\beta)}\left(1+q_{1} z+q_{2} z^{2}+\ldots\right)\left(z^{p}+\sum_{k=1}^{\infty} \phi_{p}^{\mu}(k) a_{p+k} z^{p+k}\right)
\end{gathered}
$$

On comparing the coefficients of $z^{p+k}$ on both sides in the above equation,

$$
k \phi_{p}^{\mu}(k) a_{p+k}=\frac{(p-\alpha)}{(1-\beta)}\left\{q_{k}+\phi_{p}^{\mu}(1) a_{p+1} q_{k-1}+\phi_{p}^{\mu}(2) a_{p+2} q_{k-2}+\right.
$$




$$
\left.\ldots+\phi_{p}^{\mu}(k-1) a_{p+k-1} q_{1}\right\}
$$

Hence, on using the coefficient estimate $\left|q_{k}\right| \leq 2, k \geq 1$ for Caratheodory functions $q(z)[2]$, for $k \geq 1$,

$$
\begin{aligned}
\left|a_{p+k}\right| \leq & \frac{2(p-\alpha)}{k \phi_{p}^{\mu}(k)(1-\beta)}\left\{1+\phi_{p}^{\mu}(1)\left|a_{p+1}\right|+\phi_{p}^{\mu}(2)\left|a_{p+2}\right|+\right. \\
& \left.\ldots+\phi_{p}^{\mu}(k-1)\left|a_{p+k-1}\right|\right\} .
\end{aligned}
$$

At $k=1,(21)$ gives

$$
\left|a_{p+1}\right| \leq \frac{2(p-\alpha)}{1 ! \phi_{p}^{\mu}(1)(1-\beta)}
$$

which proves (19) for $k=1$, also at $k=2,(21)$ gives

$$
\left|a_{p+2}\right| \leq \frac{2(p-\alpha)}{2 ! \phi_{p}^{\mu}(2)(1-\beta)}\left\{1+\frac{2(p-\alpha)}{(1-\beta)}\right\} .
$$

Thus, (22) and (23) prove that (19) is true for $k=1,2$. Let (19) is true for $k=n$

i.e.

$$
\left|a_{p+n}\right| \leq \frac{1}{n ! \phi_{p}^{\mu}(n)} \prod_{j=1}^{n}\left(j-1+\frac{2(p-\alpha)}{(1-\beta)}\right), n \geq 1 .
$$

Now writing inequality (21) for $k=n+1$,

$$
\begin{aligned}
\left|a_{p+n+1}\right| \leq & \frac{2(p-\alpha)}{(n+1) \phi_{p}^{\mu}(n+1)(1-\beta)}\left\{1+\phi_{p}^{\mu}(1)\left|a_{p+1}\right|+\phi_{p}^{\mu}(2)\left|a_{p+2}\right|+\right. \\
& \left.\ldots+\phi_{p}^{\mu}(n)\left|a_{p+n}\right|\right\} .
\end{aligned}
$$

On applying (22), (23) and (24),

$$
\begin{aligned}
\left|a_{p+n+1}\right| \leq & \frac{2(p-\alpha)}{(n+1) \phi_{p}^{\mu}(n+1)(1-\beta)}\left\{1+\frac{2(p-\alpha)}{1 !(1-\beta)}+\right. \\
& \left.+\frac{2(p-\alpha)}{2 !(1-\beta)}\left(1+\frac{2(p-\alpha)}{(1-\beta)}\right)+\ldots+\frac{1}{n !} \prod_{j=1}^{n}\left(j-1+\frac{2(p-\alpha)}{(1-\beta)}\right)\right\}
\end{aligned}
$$

or,

$$
\left|a_{p+n+1}\right| \leq \frac{2(p-\alpha)}{(n+1) \phi_{p}^{\mu}(n+1)(1-\beta)}\left\{\frac{1}{(n-1) !}\left(1+\frac{2(p-\alpha)}{(1-\beta)}\right) \ldots\right.
$$




$$
\left.\left(n-1+\frac{2(p-\alpha)}{(1-\beta)}\right)+\frac{1}{n !} \prod_{j=1}^{n}\left(j-1+\frac{2(p-\alpha)}{(1-\beta)}\right)\right\}
$$

or,

$$
\begin{array}{r}
\left|a_{p+n+1}\right| \leq \frac{1}{(n+1) ! \phi_{p}^{\mu}(n+1)}\left\{\frac{2(p-\alpha)}{(1-\beta)}\left(1+\frac{2(p-\alpha)}{(1-\beta)}\right) \ldots\right. \\
\left.\left(n-1+\frac{2(p-\alpha)}{(1-\beta)}\right)\left(n+\frac{2(p-\alpha)}{(1-\beta)}\right)\right\}
\end{array}
$$

or,

$$
\left|a_{p+n+1}\right| \leq \frac{1}{\phi_{p}^{\mu}(n+1)(n+1) !} \prod_{j=1}^{n+1}\left(j-1+\frac{2(p-\alpha)}{(1-\beta)}\right), n \geq 1,
$$

which shows that, the result is true for $k=n+1$. Thus by mathematical induction, (19) holds true for any $n \geq 1$. This proves the result.

Taking $\mu=0, p=1$ and $\mu=1, p=1$ respectively in Theorem 3, following results of Owa et al. [5] are obtained.

Corollary 4. [5] Let $f(z) \in S$ of the form (1) be in the family $\beta$ $U S T(\alpha), \beta \geq 0,0 \leq \alpha<1$, then

$$
\left|a_{1+k}\right| \leq \frac{1}{k !} \prod_{j=1}^{k}\left(j-1+\frac{2(1-\alpha)}{(1-\beta)}\right), k \geq 1
$$

Corollary 5. [5] Let $f(z) \in S$ of the form (1) be in the family $\beta$ $U C V(\alpha), \beta \geq 0,0 \leq \alpha<1$, then

$$
\left|a_{1+k}\right| \leq \frac{1}{(k+1) !} \prod_{j=1}^{k}\left(j-1+\frac{2(1-\alpha)}{(1-\beta)}\right), k \geq 1
$$

Again, taking $\beta=0$ in Corollary 5, following results of Robertson [6] are obtained.

Corollary 6. [6] Let $f(z) \in S$ of the form (1) be in the family $S^{*}(\alpha), 0 \leq$ $\alpha<1$, then

$$
\left|a_{1+k}\right| \leq \frac{1}{k !} \prod_{j=1}^{k}(j+1-2 \alpha), k \geq 1
$$


Corollary 7. [6] Let $f(z) \in S$ of the form (1) be in the family $K(\alpha), 0 \leq$ $\alpha<1$, then

$$
\left|a_{1+k}\right| \leq \frac{1}{(k+1) !} \prod_{j=1}^{k}(j+1-2 \alpha), k \geq 1 .
$$

Further, taking $\mu=0, \beta=0$ and $\mu=1, \beta=0$ respectively in Theorem 3, following results of Goyal and Bhagtani [3] are obtained.

Corollary 8. [3] Let $f(z) \in A(p)$ of the form (1) be in the family $S^{*}(p, \alpha), 0 \leq \alpha<p$, then

$$
\left|a_{p+k}\right| \leq \frac{1}{k !} \prod_{j=1}^{k}(j-1+2 p-2 \alpha), k \geq 1 .
$$

Corollary 9. [3] Let $f(z) \in A(p)$ of the form (1) be in the family $K(p, \alpha), 0 \leq \alpha<p$, then

$$
\left|a_{p+k}\right| \leq \frac{1}{(p+k) k !} \prod_{j=1}^{k}(j-1+2 p-2 \alpha), k \geq 1 .
$$

\section{Growth Theorem}

In this section, growth result of $f(z) \in S(\alpha, \beta, \mu, p)$ using coefficient estimate obtained in Theorem 3 and Carlson Shaffer type operator defined in (8) is proved.

Theorem 10. Let $f(z) \in A(p)$ of the form (1) be in the family $S(\alpha, \beta, \mu, p)$, then for $|z|=r<1$,

$$
|f(z)| \leq L_{p}(p+1-\mu, p+1)\left(\frac{r^{p}}{(1-r)^{\frac{2(p-\alpha)}{(1-\beta)}}}\right) .
$$

Proof. Since $f \in A(p)$ of the from (1) be in the family $S(\alpha, \beta, \mu, p)$, Theorem 3 gives

$$
\left|a_{p+k}\right| \leq \frac{\left(\frac{2(p-\alpha)}{(1-\beta)}\right)_{k}(p+1-\mu)_{k}}{(1)_{k}(p+1)_{k}} .
$$


Thus,

$$
\begin{aligned}
|f(z)| & \leq|z|^{p}+\sum_{k=1}^{\infty}\left|a_{p+k}\right||z|^{p+k} \\
& \leq|z|^{p}+\sum_{k=1}^{\infty} \frac{\left(\frac{2(p-\alpha)}{(1-\beta)}\right)_{k}(p+1-\mu)_{k}}{(1)_{k}(p+1)_{k}}|z|^{p+k} \\
& =\sum_{k=0}^{\infty} \frac{\left(\frac{2(p-\alpha)}{(1-\beta)}\right)_{k}(p+1-\mu)_{k}}{(1)_{k}(p+1)_{k}}|z|^{p+k} \\
& =\left\{\sum_{k=0}^{\infty} \frac{(p+1-\mu)_{k}}{(p+1)_{k}}|z|^{p+k}\right\} *\left\{\sum_{k=0}^{\infty} \frac{\left(\frac{2(p-\alpha)}{(1-\beta)}\right)_{k}}{(1)_{k}}|z|^{p+k}\right\} \\
& =\phi_{p}(p+1-\mu, p+1,|z|) * \phi_{p}\left(\frac{2(p-\alpha)}{(1-\beta)}, 1,|z|\right) \\
& =\phi_{p}(p+1-\mu, p+1,|z|) * \frac{|z|^{p}}{(1-\mid z)^{\frac{2(p-\alpha)}{(1-\beta)}}} \\
& =L_{p}(p+1-\mu, p+1)\left(\frac{r^{p}}{(1-r)^{\frac{2(p-\alpha)}{(1-\beta)}}} \cdot\right.
\end{aligned}
$$

This proves Theorem 10 .

For $\mu=0, \beta=0, p=1$ and $\mu=1, \beta=0, p=1$ respectively in Theorem 10, following results of Banerji and Shenan [1] are obtained.

Corollary 11. [1] Let $f(z) \in S$ be in the family $S^{*}(\alpha)$, then

$$
|f(z)| \leq \frac{r}{(1-r)^{2(1-\alpha)}},|z|=r<1
$$

Corollary 12. [1] Let $f(z) \in S$ be in the family $K(\alpha)$, then

$$
|f(z)| \leq \phi(2(1-\alpha), 2 ; r),|z|=r<1 \text {. }
$$

\section{Partial Sums}

In this section, inequalities involving partial sums of $f(z) \in A(p)$ are obtained. Let non-zero partial sums of $f(z) \in A(p)$ of the form (1) be defined as follows: 


$$
f_{0}(z)=z^{p} \text { and } f_{n}(z)=z^{p}+\sum_{k=1}^{n} a_{p+k} z^{p+k}, k \geq 1 .
$$

Theorem 13. Let $f(z) \in A(p)$ of the form (1) satisfies

$$
\sum_{k=1}^{\infty} c_{p+k}\left|a_{p+k}\right| \phi_{p}^{\mu}(k) \leq 1
$$

where $c_{p+k}:=\frac{\{k(1+\beta)+(p-\alpha)\}}{(p-\alpha)} \frac{(p+1)_{k}}{(p+1-\mu)_{k}}, \beta \geq 0,0 \leq \alpha<p, 0 \leq \mu \leq 1$, then $f \in S(\alpha, \beta, \mu, p)$ and

$$
\begin{gathered}
\operatorname{Re}\left\{\frac{f(z)}{f_{n}(z)}\right\}>1-\frac{1}{c_{p+n+1}}, \\
\operatorname{Re}\left\{\frac{f_{n}(z)}{f(z)}\right\}>\frac{c_{p+n+1}}{1+c_{p+n+1}}, \\
\operatorname{Re}\left\{\frac{f^{\prime}(z)}{f_{n}^{\prime}(z)}\right\}>1-\frac{p+n+1}{c_{p+n+1}} .
\end{gathered}
$$

Proof. Since $f(z) \in A(p)$ of the form (1) satisfy (27), from Theorem 1, $f(z) \in S(\alpha, \beta, \mu, p)$. Further, from (27), as it is easily seen that

$$
\begin{aligned}
c_{p+n+1}>c_{p+n} & >1 \\
\sum_{k=1}^{n}\left|a_{p+k}\right|+c_{p+n+1} \sum_{k=n+1}^{\infty}\left|a_{p+k}\right| & \leq \sum_{k=1}^{\infty} c_{p+k}\left|a_{p+k}\right| \leq 1 .
\end{aligned}
$$

Set

$$
g_{1}(z)=c_{p+n+1}\left\{\frac{f(z)}{f_{n}(z)}-\left(1-\frac{1}{c_{p+n+1}}\right)\right\}
$$

which is analytic in $U$ and $g_{1}(0)=1$.

$$
\begin{aligned}
\left|\frac{g_{1}(z)-1}{g_{1}(z)+1}\right| & =\left|\frac{c_{p+n+1} \sum_{k=n+1}^{\infty} a_{p+k} z^{k}}{2+2 \sum_{k=1}^{n} a_{p+k} z^{k}+c_{p+n+1} \sum_{k=n+1}^{\infty} a_{p+k} z^{k}}\right| \\
& \leq \frac{c_{p+n+1} \sum_{k=n+1}^{\infty}\left|a_{p+k}\right|}{2-2 \sum_{k=1}^{n}\left|a_{p+k}\right|-c_{p+n+1} \sum_{k=n+1}^{\infty}\left|a_{p+k}\right|} \\
& \leq 1
\end{aligned}
$$

if (32) holds, which readily yields that $\operatorname{Re}\left\{g_{1}(z)\right\}>0$, this proves assertion (28) of Theorem 10. 
Similarly, set

$$
\begin{aligned}
g_{2}(z) & =\left(1+c_{p+n+1}\right)\left\{\frac{f_{n}(z)}{f(z)}-\frac{c_{p+n+1}}{1+c_{p+n+1}}\right\} \\
& =\left\{1-\frac{\left(1+c_{p+n+1}\right) \sum_{k=n+1}^{\infty} a_{p+k} z^{k}}{1+\sum_{k=1}^{\infty} a_{p+k} z^{k}}\right\}
\end{aligned}
$$

and making use of (32),

$$
\begin{aligned}
\left|\frac{g_{2}(z)-1}{g_{2}(z)+1}\right| & =\left|\frac{\left(1+c_{p+n+1}\right) \sum_{k=n+1}^{\infty} a_{p+k} z^{k}}{2+2 \sum_{k=1}^{\infty} a_{p+k} z^{k}-\left(1+c_{p+n+1}\right) \sum_{k=n+1}^{\infty} a_{p+k} z^{k}}\right| \\
& \leq \frac{\left(1+c_{p+n+1}\right) \sum_{k=n+1}^{\infty}\left|a_{p+k}\right|}{2-2 \sum_{k=1}^{n}\left|a_{p+k}\right|-\left(c_{p+n+1}-1\right) \sum_{k=n+1}^{\infty}\left|a_{p+k}\right|} \\
& \leq 1
\end{aligned}
$$

which proves the assertion (29).

Further, set

$$
\begin{gathered}
g_{3}(z)=\frac{c_{p+n+1}}{p+n+1}\left\{\frac{f^{\prime}(z)}{f_{n}^{\prime}(z)}-\left(1-\frac{p+n+1}{c_{p+n+1}}\right)\right\} \\
=1+\frac{\frac{c_{p+n+1}}{p+n+1} \sum_{k=n+1}^{\infty} \frac{(p+k)}{p} a_{p+k} z^{k}}{1+\sum_{k=1}^{n} \frac{(p+k)}{k} a_{p+k} z^{k}} . \\
\left|\frac{g_{3}(z)-1}{g_{3}(z)+1}\right|=\left|\frac{\frac{c_{p+n+1}}{p+n+1} \sum_{k=n+1}^{\infty} \frac{(p+k)}{p} a_{p+k} z^{k}}{2+2 \sum_{k=1}^{n} \frac{(p+k)}{p} a_{p+k} z^{k}+\frac{c_{p+n+1}}{p+n+1} \sum_{k=n+1}^{\infty} \frac{(p+k)}{p} a_{p+k} z^{k}}\right| \\
\leq \frac{\frac{c_{p+n+1}}{p+n+1} \sum_{k=n+1}^{\infty} \frac{(p+k)}{p}\left|a_{p+k}\right|}{2-2 \sum_{k=1}^{n} \frac{(p+k)}{p}\left|a_{p+k}\right|-\frac{c_{p+n+1}}{p+n+1} \sum_{k=n+1}^{\infty} \frac{(p+k)}{p}\left|a_{p+k}\right|} \leq 1
\end{gathered}
$$

if

$$
\sum_{k=1}^{n} \frac{(p+k)}{p}\left|a_{p+k}\right|+\frac{c_{p+n+1}}{p+n+1} \sum_{k=n+1}^{\infty} \frac{(p+k)}{p}\left|a_{p+k}\right| \leq 1
$$

which holds if the left hand side of (37) is bounded above by $\sum_{k=1}^{\infty} c_{p+k}\left|a_{p+k}\right|$ i.e. if

$$
\sum_{k=1}^{n}\left(c_{p+k}-\frac{(p+k)}{p}\right)\left|a_{p+k}\right|
$$




$$
+\sum_{k=n+1}^{\infty}\left(c_{p+k}-\frac{c_{p+n+1}}{p+n+1} \frac{(p+k)}{p}\right)\left|a_{p+k}\right| \geq 0
$$

As $\frac{c_{p+k}}{p+k}$ is an increasing function of $k,(38)$ is true. Thus $\operatorname{Re}\left\{g_{3}(z)\right\}>0$ which readily yields the assertion (30).

Remark 14. Note that taking $\beta=1, p=1$ and $\mu=0, \mu=1$ respectively, above results of Theorem 13 coincide with results obtained by Rosy [7].

\section{Integral Mean Inequality}

The following subordination result due to Littlewood [4] is used in next Theorem.

Lemma 15. [4] Let $f(z)$ and $g(z)$ be analytic in $U$ with $f(z) \prec g(z)$, then

$$
\int_{0}^{2 \pi}\left|f\left(r e^{i \theta}\right)\right|^{q} d \theta \leq \int_{0}^{2 \pi}\left|g\left(r e^{i \theta}\right)\right|^{q} d \theta
$$

where $q>0, z=r e^{i \theta}$ and $0<r<1$.

Theorem 16. Let $f(z) \in T(p)$ of the form (2) be in the family $T S(\alpha, \beta, \mu, p)$, then for $z=r e^{i \theta}, 0<r<1$ and $q>0$

$$
\int_{0}^{2 \pi}\left|f\left(r e^{i \theta}\right)\right|^{q} d \theta \leq \int_{0}^{2 \pi}\left|f_{1}\left(r e^{i \theta}\right)\right|^{q} d \theta
$$

where

$$
f_{1}(z)=z^{p}-\frac{(p-\alpha)(p+1-\mu)}{\{(1+\beta)+(p-\alpha)\}(p+1)} z^{p+1} .
$$

Proof. For

$$
f(z)=z^{p}-\sum_{k=1}^{\infty}\left|a_{p+k}\right| z^{p+k}
$$

and $f_{1}(z)$ given by (41), to prove (40), it is equivalent to show

$$
\int_{0}^{2 \pi}\left|1-\sum_{k=1}^{\infty}\right| a_{p+k}\left|z^{k}\right|^{q} d \theta \leq \int_{0}^{2 \pi}\left|1-\frac{(p-\alpha)(p+1-\mu)}{\{(1+\beta)+(p-\alpha)\}(p+1)} z\right|^{q} d \theta .
$$


Hence, by Lemma 15, it suffices to show

$$
1-\sum_{k=1}^{\infty}\left|a_{p+k}\right| z^{k} \prec 1-\frac{(p-\alpha)(p+1-\mu)}{\{(1+\beta)+(p-\alpha)\}(p+1)} z
$$

which is true if there exists a Schwartz function $w(z)$ such that

$$
1-\sum_{k=1}^{\infty}\left|a_{p+k}\right| z^{k}=1-\frac{(p-\alpha)(p+1-\mu)}{\{(1+\beta)+(p-\alpha)\}(p+1)} w(z) .
$$

Using (18) of Theorem 2,

$$
\begin{aligned}
|w(z)| & =\left|\frac{\{(1+\beta)+(p-\alpha)\} \phi_{p}^{\mu}(1)}{(p-\alpha)} \sum_{k=1}^{\infty}\right| a_{p+k}\left|z^{k}\right| \\
& \leq|z| \\
& <1 .
\end{aligned}
$$

This completes the proof of Theorem 16.

\section{References}

[1] P.K. Banerji, G.M. Shenan, Application of fractional differintegral operators to certain family of analytic functions with positive coefficients, Bull. Cal. Math. Soc., 95, No. 5 (2003), 377-384.

[2] C. Carathéodory, Über den Variabilitatsbereich der Fourier'schen Konstanten Von Possitiven Harmonischen Funktionen, Rend. Circ. Palermo., 32 (1911), 193-217.

[3] S.P. Goyal, M. Bhagtani, Distrotion inequalities for the Dziok Srivastava linear operator involving the generalized hypergeometric function, Tamsui Oxford J. Math. Sci., 24, No. 3 (2008), 215-223.

[4] J.E. Littlewood, On inequalities in the theory of functions, Proc. London Math. Soc., 23 (1925), 481-519.

[5] S. Owa,Y. Polatoglu, E. Yavuz, Coefficient inequalities for classes of uniformly starlike and convex functions, J. Ineq. in Pure and Appl. Math., 7, No. 5 (2006), Art. 160. 
[6] M.S. Robertson, On the theory of univalent functions, Annals of Math., 37 (1936), 374-408.

[7] T. Rosy, Studies on Subclasses of Starlike and Convex Functions, Ph.D. Thesis, Madras University (2001).

[8] H. Saitoh, A linear operator and its application of first order differential subordination, Math. Japan, 44 (1996), 31-38.

[9] H.M. Srivastava, S. Owa (Ed-s.), Current Topics in Analytic Function Theory, World Scientific Publication, Singapore (1992). 
